\title{
Effektivität standortbezogener Werbung im digitalen Marketing
}

\author{
Martin Spann*
}

Ausgangslage

Mobile Werbung ist, gemessen an den Werbeausgaben, inzwischen der wichtigste digitale Werbekanal (Koetsier 2018). Über mobile Endgeräte können Verbraucher in Echtzeit angesprochen und die Werbung zielgerichtet auf den Standort des Verbrauchers angepasst werden. Geografische Nähe zwischen Konsument und dem Standort eines Geschäfts erhöht die Relevanz für die Verbraucher und damit die Wirksamkeit einer Werbekampagne.

Im Rahmen eines deutschlandweiten Feldexperiments mit 3.499 verschiedenen Kampagnen für 3.930 verschiedene Geschäfte wurden die Wirksamkeit standortbezogener Werbung sowie unterschiedliche Ausgestaltungsformen für die Darstellung bzw. das (App) Design getestet (Molitor et al. 2020).

Die Ausgangslage ist das sogenannte Pull-Mobile-Advertising, bei dem Konsumenten proaktiv standortbezogene Werbung anfordern und bezogen auf ihren aktuellen Standort angezeigt bekommen. Zwei wichtige Ausgestaltungsformen sind dabei i) die Sortierung nach geographischer Distanz zwischen Werbetreibenden (z.B. Geschäft) und Konsument (i.d.R. werden nähere Geschäfte zuerst angezeigt) und ii) die Bereitstellung der genauen Distanzinformation. Beide Dimensionen wurden experimentell variiert (siehe Abbildung 1): neben einer Sortierung nach Distanz wurden die Werbeanzeigen zufällig sortiert, wobei die genaue Entfernung zwischen Verbraucher und Geschäft entweder gezeigt oder weggelassen wurde.

Prof. Dr. Martin Spann | Institut für Electronic Commerce und Digitale Märkte | LudwigMaximilians-Universität München | spann@spann.de 


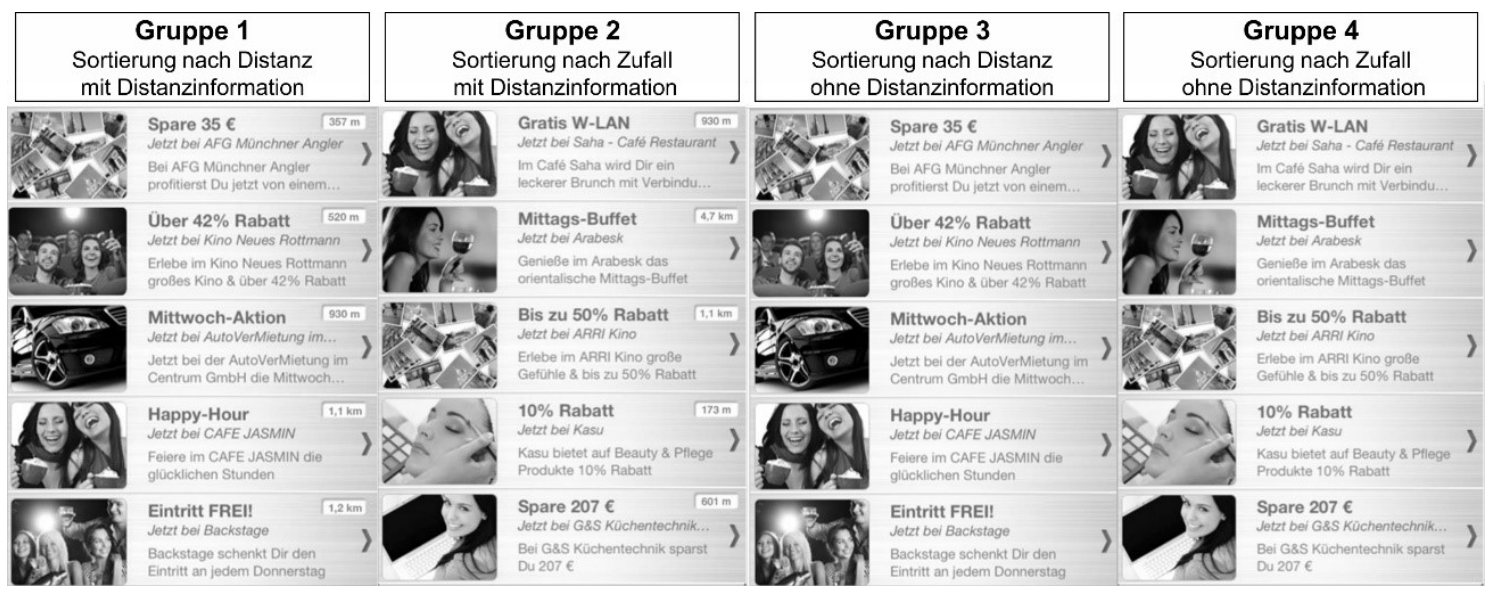

Abb. 1: $\quad$ Ausgestaltung der experimentellen Gruppen. Molitor et al. 2020: 439.

\section{Wesentliche Ergebnisse}

Standortbezogene Werbung ist effektiv: Bezogen auf eine Nutzung (Session) der App lag die durchschnittliche Reaktionsrate der Konsumenten in der Studie bei 16,38 \% und auf Ebene der einzelnen Werbeanzeige bei 1,35 \%. Die Effektivität variiert über Produktkategorien: Bei Lebensmitteln liegt die Effektivität mit 1,98 \% um $47 \%$ höher als die durchschnittliche Effektivität.

Je kürzer die Distanz zwischen Verbraucher und Geschäft, desto höher ist die Effektivität.

Eine Sortierung nach Distanz (jeweils naheste Geschäfte zuerst) erhöht die Effektivität um $21 \%$, wohingegen das Anzeigen der genauen Distanzinformation keinen nennenswerten Einfluss auf die Effektivität hat (Anzeige der genauen Distanzinformation erhöht Effektivität nur um <1 \%). Letzteres kann auch dadurch erklärt werden, dass Konsumenten eine generelle Vorstellung bezüglich der ungefähren Distanzen zu Geschäften haben.

Die Effektivität standortbezogener Werbung ist höher im ländlichen Raum und in Vorstädten als im innerstätischen Bereich, was durch eine insgesamt geringere Anzahl an Geschäften und dadurch eine höhere Toleranz für Entfernungen zu Geschäften im ländlichen Raum erklärt werden kann.

\section{Implikationen für Unternehmen}

Standortbezogene Werbung ist für ein breites Spektrum von Produktkategorien effektiv und ein relevantes Werbeinstrument für lokale Geschäfte (Handel und Dienstleistungen). Die Effektivität standortbezogener Werbung ist im ländlichen und vorstädtischen Raum noch höher als in den Innenstädten. 
Bei der Anzeige standortbezogener Werbung sollte eine Sortierung nach Distanz verwendet werden, wobei aber innerhalb von Entfernungsbereichen (z.B. 0-1 km und 1-2 km) auch nach anderen Kriterien sortiert werden könnte. Die Anzeige der exakten geographischen Distanz ist nicht erforderlich.

Innerhalb von Entfernungsbereichen können Anbieter von standortbezogener Werbung einzelne Anzeigeplätze anhand eines Auktionsmechanismus versteigern. Außerdem können sich die Werbepreise nach Standort unterscheiden (z. B. städtische Gebiete gegenüber ländlichem Raum).

Geschäfte (Werbetreibende) können standortbezogene Werbung auch nutzen, um dynamische Preisgestaltung in Kombination mit standortbezogener Werbung anzuwenden. Beispielsweise ist es denkbar den Angebotspreis (bzw. Rabatthöhe) je nach Distanz des Verbrauchers zum Geschäft anzupassen, um bei größeren Distanzen Konsumenten durch attraktivere Angebote zum Geschäftsbesuch zu bewegen. Darüber hinaus können Informationen zur Effektivität standortbezogener Werbung zur Kampagnenplanung und Sortimentsgestaltung in lokalen Geschäften genutzt werden.

\section{Literaturverzeichnis}

Koetsier, J. 2018. Mobile Advertising Will Drive 75\% Of All Digital Ad Spend In 2018: Here's What's Changing. [online] https://www.forbes.com/sites/johnkoetsier/2018/02/23/mobileadvertising-will-drive-75-of-all-digital-ad-spend-in-2018-heres-whatschanging.

Molitor, D., M. Spann, A. Ghose, P. Reichhart. 2020. Effectiveness of Location-Based Advertising and the Impact of Interface Design. Journal of Management Information Systems 37: 431-456.

\section{Quelle}

Der Beitrag basiert auf "Effectiveness of Location-Based Advertising and the Impact of Interface Design" von Dominik Molitor, Martin Spann, Anindya Ghose und Philipp Reichhart erschienen in: Journal of Management Information Systems, 37. Jg. 2020, S. 431-456. 